\title{
Targeted and personalized therapy for cancer: Theory and practice in China
}

\author{
LIU Qiang $^{1,2}$, ZHANG XiaoShi $^{1,3}$ \& Zeng YiXin ${ }^{1,2,4^{*}}$ \\ ${ }^{1}$ State Key Laboratory of Oncology in South China, Guangzhou, 510060, China; \\ ${ }^{2}$ Experimental Research Department, Sun Yat-sen University, Guangzhou 510060, China; \\ ${ }^{3}$ Cancer Biotherapy Center, Guangzhou 510060, China; \\ ${ }^{4}$ Chinese Academy of Medical Sciences and Peking Union Medical College, Beijing 100005, China
}

Received October 10, 2011, accepted November 9, 2011

Citation: Liu Q, Zhang X S, Zeng Y X. Targeted and personalized therapy for cancer: Theory and practice in China. Sci China Life Sci, 2011, 54: 1081-1084, doi: $10.1007 / \mathrm{s} 11427-011-4253-\mathrm{Z}$

Molecular targeted therapy (MTT), using chemicals of low molecular weight, monoclonal antibodies, and/or polypeptides to interfere with specific signaling pathways in cancer cells, causes inhibition of tumor progression. Clinical evidence has demonstrated that MTT has promising potential in not only killing tumors but also inducing tumor cells to differentiate into normal cells, leading to cure of the patients. Furthermore, MTT leads to potent inhibition of oncogenic signals. The delay of tumor progression makes the patients "survive with tumor". Thus, in the future it is possible that cancer becomes a chronic disease much like diabetes and hypertension. This article reviews the theory on molecular targeted therapy, its features and future direction, and contributions in the field from Chinese scientists.

\section{The concept of molecular targeted therapy}

In the last two centuries, there were two leaps in the treatment of malignant tumors. One was in 1890 when Halsted proposed the concept of radical surgery for cancer, and the other was in the 1970s when Fish integrated the chemical treatment with radical surgery (adjuvant chemotherapy or neoadjuvant chemotherapy). Since then, the treatment of malignant tumor did not have much development until the emergence of molecular targeted drugs. In the late 20th

*Corresponding author (email: zengyix@ mail.sysu.edu.cn) century, researchers began to study tumor in a system biological point of view and realized that tumor is a multi-factor participated, multi-step in development and systemic disease. Mutation of oncogenes, missing of tumor suppressor genes or abnormal gene expressions often leads to genomic instability in tumor cells and further abnormal biological behaviors in tumor cells, such as (i) self-sufficiency in growth signals, (ii) insensitivity to growth inhibition signals, (iii) evading apoptosis, (iv) unlimited potential for replication, (v) angiogenesis, (vi) invasion and metastasis, and (vii) immune escape. Molecular targeted therapy refers to the use of chemicals of low molecular weight, monoclonal antibodies, polypeptides and other substances to interfere with specific signaling pathways that regulate biological behaviors of tumor cells, thereby inhibiting tumor progression. Molecular targeted therapy is essentially different from traditional chemotherapy, in which small molecular compounds are used to directly damage DNA, impede cell mitosis or induce cell death. The drawback is that chemotherapy lacks selectivity between normal cells and tumor cells. Drug toxicity and side effects limit the intensity and frequency of chemotherapy. It harms not only the function of vital organs such as heart, liver, lung, kidney, bone marrow, but also immune system, resulting in the loss of self-protective barriers to tumor. In addition, chemotherapy increases the instability of the tumor cell genome, which will precipitate the process for tumor cells to develop adap- 
tation to chemotherapeutic drugs. Therefore, toxicity and side effects of chemotherapy and drug resistance may make it difficult for most patients to accomplish chemotherapy. In contrast, molecular targeted therapy targets abnormal signaling pathways in tumor cells. With virtues of high selectivity, low toxicity and high therapeutic index, molecular targeted therapy can be used for a long time, which then may help transform malignant tumors into a chronic disease similar to hypertension or diabetes.

Starting from 1997 when the monoclonal antibody trastuzumab (Herceptin) and rituximab (MabThera) were approved for the treatment of metastatic breast cancer and diffuse large B-cell lymphoma, the history of molecular targeted therapy is merely longer than a decade. So far, more than ten kinds of molecular targeted drugs have been approved for the treatment of solid tumors, and some others for hematological malignancies. Patient survival time is the gold standard for evaluating drug efficacy. The value of molecular targeted therapy is demonstrated in two ways: on the one hand, through inducing tumor cell differentiation or combining with surgery, radiotherapy, chemotherapy and other treatment measures, molecular-targeted drugs are able to increase 5-year survival rate of patients with neoplasm, in other words, more patients will be cured. Examples are trans-retinoic acid (acute promyelocytic leukemia), trastuzumab (breast cancer), rituximab (lymphoma) and imatinib (gastrointestinal stromal tumors, chronic myelogenous leukemia), etc. On the other hand, by delaying tumor progression molecular targeted therapy will improve the quality of life of patients and extend their survival time. Examples are erlotinib (non-small cell lung cancer, pancreatic cancer), gefitinib (non-small cell lung cancer), temsirolimus (renal cell carcinoma), bevacizumab (colorectal cancer, non-small cell lung cancer, breast cancer and renal cell carcinoma), cetuximab (colorectal carcinoma, head and neck squamous cell carcinoma), apatinib (breast cancer) and sorafenil (renal cell carcinoma), sunitinib (renal cell carcinoma, gastrointestinal stromal tumors), endostar (non-small cell lung cancer) and bortezomib (multiple myeloma) and so on. With the further research and development, the clinical value of molecular targeted therapy will be constantly enriched and expanded.

\section{Featured achievements of molecular targeted therapy}

\subsection{Induction of tumor cells to differentiate into nor- mal cells for curing the patients}

Compared with normal cells, tumor cells have one different yet important biological feature-poor differentiation, thus neoplasm is seen as a differentiation disorder or a dedifferentiation disease. Differentiation-induction is described as inducing redifferentiation of malignant cells into normal or nearly normal cells for making changes to their malignant biological behaviors, stopping their vicious hyperplasia, and ultimately leading to alleviation of malignant tumors or even cure of them without damaging normal cells. Compared with traditional treatment choices of surgery, chemotherapy, radiotherapy and others based on the concept of "irreversible tumor cells", differentiation-induction has opened up a new door for the treatment for malignant tumors, with the example drug being trans-retinoic acid. Since1980, medical scientists in China, as Drs. Wang ZhenYi and Chen Zhu, have successfully developed retinoic acid (RA) which may induce tumor cells to differentiate into normal hematopoietic cells, with eastern philosophy's concept of "Dayu's method of controlling the flood instead of stopping the flood" as their guidance. It is a milestone for tumor differentiation-induction therapy and a model of tumor targeted therapy. On the basis of in-depth study of the role and mechanism of RA and RA-like substance in the prevention and treatment of tumor, the application of all-trans retinoic acid (ATRA) in the treatment of acute promyelocytic leukemia (APL) has increased the complete remission rate to $87 \%-92 \%$ according to the latest data, showing a promising prospect in tumor treatment and a new way created of leukemia therapy. APL is the first human malignant tumor cured for its tumor-specific biological behavior. More than $95 \%$ of APL patients have the characteristic non-random chromosome $\mathrm{t}(15 ; 17)$. The translocation helps promyelocytic leukemia (PML) gene on chromosome 15 to be fused with the retinoic acid receptor $\alpha$ (RAR- $\alpha$ ) gene on chromosome 17. The expression of the PML-RAR fusion protein is crucial to the mechanism of differentiation block and onset of APL. Targeting against this, all-trans retinoic acid may dismiss the differentiation block effect of PML-RAR protein and propel differentiation of leukemia cells to normal cells.

\subsection{Inhibition of abnormal oncogenic signaling of tu- mor cells to suppress tumor growth and let the patient "live with tumor"}

Abnormal activation of oncogenes is a main propeller in tumor development, and decrease of its power will delay tumor progression. Chronic myelogenous leukemia (CML) is characterized by Philadelphia chromosome $\left(\mathrm{Ph}^{+}\right)$with more than $90 \%$ of all CML cases have the marker chromosome- $\mathrm{Ph}^{+}$chromosome. Molecular basis for this disease is that the translocation between the abl gene on chromosome 9 and the bcr gene on chromosome 22 forms bcr/abl fusion gene which expresses the bcr-abl fusion protein-tyrosine kinase. Imatinib mesylate (Gleevec) is a tyrosine kinase inhibitor selectively inhibiting the tyrosine kinase produced by bcr/abl fusion gene. As the first human tyrosine kinase inhibitor, Gleevec is very effective in treating CML and the $\mathrm{CR}$ rate for newly diagnosed $\mathrm{Ph}^{+}$ALL is up to $95 \%$. Imatinib is important in history because its emergence verified the "oncogene" hypothesis. Under the guidance of this 
drug-developing strategy, many tyrosine kinase inhibitors for different receptors, including EGFR, Her2, PDGFR, have been developed. Among them, trastuzumab (Herceptin), a specific Her2 antibody, is another outstanding drug proving once again that "oncogene" is very important in the occurrence and development of solid tumors as well. Her2/neu (erb-B-2), expressed in 20\%-30\% of metastatic breast cancer, predicts poor prognosis. After trastuzumab binds with the receptor, the corresponding receptor tyrosine kinase signaling will be blocked, thus inhibiting tumor cell growth and tumor angiogenesis and increasing tumor cell sensitivity to apoptosis signals. The antibody-dependent cytotoxicity (ADCC) may also be one of the mechanisms by which trastuzumab works.

No matter trastuzumab is used as monotherapy or in combined chemotherapy, this drug can significantly inhibit the development of breast cancer with high Her2 expression (++-+++). More importantly, a number of studies have confirmed that trastuzumab used as adjuvant therapy can significantly reduce tumor recurrence and mortality rate in Her2-positive patients.

\subsection{Enhancing anti-tumor effect with both intervention on tumor stroma and inhibition of tumor cells acting together}

Tumor stroma is composed of normal cells and their products. The genome of stromal cells is stable and it is not so easy for them to develop drug-resistance, making them one of the ideal targets of anti-tumor therapy. Although as early as 1974, Folkman proposed to inhibit tumor growth by inhibiting tumor angiogenesis, the idea of anti-angiogenisis was not accepted by the public until 1997 when endostatin, a specific inhibitor of angiogenesis, was found. In the year of 2004 a VEGF-specific monoclonal antibody bevacizumab came into market, demonstrating anti-angiogenesis strategy was finally turned into reality. Clinical studies have shown that only inhibition of tumor angiogenesis itself doesn't have strong tumor-inhibitory effect, suggesting tumor cells are in dominant position compared with neovascularization. So a two-phase treatment strategy to inhibit both tumor cells and tumor blood vessels was developed. Tumor angiogenesis inhibitors have proved that tumor stroma plays an important role in the tumor development and interference with tumor stroma is an important complement to the strategy of directly suppression of tumor cells. In the near future, the tumor interstitial matrix or other cells in the stroma such as lymphocytes, macrophages, and fibroblasts are likely to become targets for treatment.

\subsection{Application of the immunological characteristics of antibody in specifically killing tumor cells}

The specific binding of antibody and antigen lays basis for a highly selective treatment. A number of clinical trials have proven that rituximab, a CD20-specific antibody, when used in combined chemotherapy, can significantly improve the prognosis of diffuse large B cell lymphoma and low-grade follicular malignant lymphoma. CD20 is a lineage marker instead of an "oncogene". The success of rituximab shows that with the guidance of lineage marker, monoclonal antibodies may make use of the immunological characteristics of antibody, such as ADCC and complement-dependent cytotoxicity to inhibit tumor growth. Based on the guiding role of antibodies, radiation immunology has been developed and several monoclonal antibodies used in radiation for lymphoma, liver cancer, lung cancer have already come into market.

\section{Problems and trends of molecular targeted therapy}

\subsection{Looking for targets more specific to tumor cells}

At present, the nature of tumor is still not clear. Oncogenes/tumor suppressor genes control the occurrence and development of malignant tumors, but they are also involved in important physiological activities. How to moderately interfere with these oncogenic signaling remains a huge challenge. In recent years, a cancer stem cell hypothesis provides us with new understanding for the mechanism of occurrence, development and metastasis of malignant tumors. It now appears that the vast majority of tumor cells in most types of malignant tumors have a certain degree of sensitivity to conventional treatments such as radiotherapy and chemotherapy, only a small number of tumor cells have superb self-replicate ability and the ability to pump out chemotherapy drugs and thus developing drug resistance. Such cells are called cancer stem cells. The first studies in hematology have confirmed that not only the existence of cancer stem cells is a cause for the resistance, recurrence and metastasis, but also malignant transformation of stem cells at different stages is a cause for tumor occurrence. Our laboratory has recently isolated some tumor cells with phenotypic characteristics of stem cells from nasopharyngeal carcinoma, and confirmed that these cells do have abilities of anti-radiation, developing drug-resistance and metastasis much stronger than other tumor cells. Are these tumor cells with phenotypic characteristics of stem cells a manifestation of heterogeneity in tumor cells? We all know that loss of genomic stability is an essential feature of tumor cells, and that it will lead to a high degree of heterogeneity in tumor cells. So when we examine tumor cells in the pathological slices, we can see a great variety in the number of chromosomes and a high frequency of chromosome fragment loss and amplification without much regularity. In view of this, we must think about (i) how to develop drugs more focused and targeting those tumor cells with the phenotype of stem cells; (ii) we should be more careful in the use of cytotoxic drugs with impact on genomic stability. 
3.2 Individualized therapy under the guidance of molecular markers to improve the effectiveness of treatment

Malignant tumors are of high heterogeneity at the molecular level. Loss of tumor genomic instability will make tumor cells evolve in unpredictable directions. The present theory from evidence-based medicine neglects the heterogeneity of tumor cells. It is of much blindness to treat individual patients under the guidance of prognosis of groups of patients and the value of molecular targeted therapy is also largely underestimated. With the help of chip technology and bioinformatics technology, we can accurately classify tumor cells in the level of gene structure and expression level (molecular typing) and use the information to guide treatment. The Cancer Center of Sun Yat-sen University has conducted a preliminary exploration in this area. As tumor development has multi-gene in participation and multi-step in progress, it is necessary to use multi-target combined treatment and multi-target sequential therapy in achieving the goal of "living with tumor". In the future it is possible to determine genome-widely one or several abnormal genetic signaling indispensable to tumor cells in some stage during the development of disease, for which accurate treatment can be used for the identified target. On the basis of "treatment according to the identified target" and heterogeneity of tumors, we can achieve "treating the apparently same diseases with different methods and treating apparently different diseases with the same method"-an old saying from Chinese Medicine describing excellent therapeutics.

In addition to "treatment according to the identified target", another key in elevating treatment accuracy is to maximize the biological effects of targeted drugs. Molecular targeted drugs are non-cytotoxic with prominent individual differences in their tolerability and plasma concentration. It is difficult to find a most appropriate effective dose between the lowest effective dose and the highest tolerated dose by conventional clinical trials. So it is necessary to undertake "individualized titration" in the course of treatment based on targets related biological response markers to maximize the efficacy of molecular targeted drugs.

A decade of efforts in pursuing our dream now seems only a flash passed. Clinical practice has proved that the molecular targeted therapy is a young and dynamic treatment strategy and drug treatment for malignant tumors is undergoing a process from quantitative change to qualitative change. For chemotherapy-sensitive tumors, molecular targeted therapy combined with traditional therapeutic means is able to further improve the prognosis of patients. For tumors insensitive to chemotherapy, molecular targeted therapy will gradually replace the chemotherapy and bring in the upcoming "post-chemotherapy era" in the near future. It is a pride for us Chinese people that in this great historical change. The contribution of Chinese scientists is really noticeable. Drs. Wang ZhenYi and Chen Zhu have been internationally recognized as the finder of the trans-retinoic acid-induced differentiation of leukemia cells. Dr. Luo YongZhang developed modified endostatin (Endostar). Dr. Chen ZhiNan developed a monoclonal antibody for liver cancer's radioimmunotherapy. Dr. Peng ZhaoHui carried out industrialization of the first gene therapy drug-recombinant human p53 adenovirus injection. Very recently, a small molecular drug, Conmana, was successfully developed by a Chinese company for targeted therapy for latestage lung cancer patients. These achievements have contributed to the development of tumor molecular targeted therapy.

Open Access This article is distributed under the terms of the Creative Commons Attribution License which permits any use, distribution, and reproduction in any medium, provided the original author(s) and source are credited. 